OPEN ACCESS

Edited by:

Peter R. Girguis,

Harvard University, United States

Reviewed by:

Jorge Reinheimer,

National University of the Littoral,

Argentina

Akira Hishinuma

Dokkyo Medical University, Japan

*Correspondence: Henrique C. P. Figueiredo figueiredoh@yahoo.com

Specialty section: This article was submitted to Aquatic Microbiology, a section of the journal Frontiers in Microbiology

Received: 17 May 2017 Accepted: 25 July 2017 Published: 09 August 2017

Citation:

Assis GBN, Pereira FL, Zegarra AU,

Tavares GC, Leal CA and

Figueiredo HCP (2017) Use of MALDI-TOF Mass Spectrometry for the Fast Identification of Gram-Positive Fish Pathogens.

Front. Microbiol. 8:1492.

doi: 10.3389/fmicb.2017.01492

\section{Use of MALDI-TOF Mass Spectrometry for the Fast Identification of Gram-Positive Fish Pathogens}

\author{
Gabriella B. N. Assis, Felipe L. Pereira, Alexandra U. Zegarra, Guilherme C. Tavares, \\ Carlos A. Leal and Henrique C. P. Figueiredo*
}

AQUACEN, National Reference Laboratory for Aquatic Animal Diseases, Ministry of Agriculture, Livestock and Food Supply, Federal University of Minas Gerais, Belo Horizonte, Brazil

Gram-positive cocci, such as Streptococcus agalactiae, Lactococcus garvieae, Streptococcus iniae, and Streptococcus dysgalactiae subsp. dysgalactiae, are found throughout the world, particularly in outbreaks in farmed fish, and are thus associated with high economic losses, especially in the cultivation of Nile Tilapia. The aim of this study was to evaluate the efficacy of matrix-assisted laser desorption ionization (MALDI)-time of flight (TOF) mass spectrometry (MS) as an alternative for the diagnosis of these pathogens. One hundred and thirty-one isolates from Brazilian outbreaks assisted by the national authority were identified using a MALDI Biotyper from Bruker Daltonics. The results showed an agreement with respect to identification (Kappa $=1)$ between this technique and $16 S$ ribosomal RNA gene sequencing for $S$. agalactiae and L. garvieae. However, for $S$. iniae and S. dysgalactiae subsp. dysgalactiae, perfect agreement was only achieved after the creation of a custom main spectra profile, as well as further comparisons with $16 \mathrm{~S}$ ribosomal RNA and multilocus sequence analysis. MALDI-TOF MS was shown to be an efficient technology for the identification of these Gram-positive pathogens, yielding a quick and precise diagnosis.

Keywords: MALDI-TOF MS, S. agalactiae, S. iniae, S. dysgalactiae subsp. dysgalactiae, Lactococcus garvieae

\section{INTRODUCTION}

Gram-positive cocci infections pose a great threat to farmed fish worldwide (Evans et al., 2002; Agnew and Barnes, 2007; Abdelsalam et al., 2013) and especially impact warm water systems used for the cultivation of Nile tilapia, one of the major commodities of global aquaculture (FAO, 2016). Four pathogens that are highly associated with outbreaks in fish farms are Streptococcus agalactiae, Lactococcus garvieae, Streptococcus iniae, and S. dysgalactiae subsp. dysgalactiae (SDD) (Evans et al., 2002; Agnew and Barnes, 2007; Mian et al., 2009; Netto et al., 2011; Figueiredo et al., 2012; Abdelsalam et al., 2013). Streptococcus agalactiae, S. iniae, and L. garvieae cause septicemia and meningoencephalitis in several species of marine and freshwater fish (Eldar et al., 1995; Evans et al., 2002; Mian et al., 2009; Figueiredo et al., 2012; Godoy et al., 2013; Soto et al., 2015; Fukushima et al., 2017). In fish, SDD infections are characterized by a systemic multifocal inflammatory reaction and a focal necrosis of the caudal peduncle, with moderate to high mortality rates during outbreaks (Nomoto et al., 2006). 
Currently, the most widely used technology for the diagnosis of these infectious diseases is the isolation of the etiological agent in blood agar medium and subsequent identification through phenotypic/biochemical tests (Vendrell et al., 2006; Figueiredo et al., 2012; Assis et al., 2016). However, the performance of these tests can lead to misidentification or a lack of species-level resolution (Brigante et al., 2006; Tavares et al., 2016). Alternative molecular methods, such as species-specific PCR (Poyart et al., 1998) and the amplification and sequencing of the $16 \mathrm{~S}$ ribosomal RNA (rRNA) gene, are useful for diagnosis (Kolbert and Persing, 1999; Patel, 2001; Clarridge, 2004) but are expensive and time consuming, mostly in trials with large number of clinical samples.

Recently, another technology to identify microorganisms was released: matrix-assisted laser desorption ionization (MALDI)time of flight (TOF) mass spectrometry (MS) (Clark et al., 2013; Singhal et al., 2015). In this technique, the identification of the bacterial species is done by a comparison of peptide mass fingerprints to the device database. A typical mass range of 2-20 $\mathrm{kDa}$ is used, which represents mainly ribosomal proteins, along with a few housekeeping proteins (Singhal et al., 2015). There are many studies demonstrating the efficiency of MALDI-TOF MS in the classification of several species in a shorter time and with a lower cost (Bilecen et al., 2015), including typing (Nagy et al., 2011; Rizzardi et al., 2013) or identification of specific markers such as methicillin resistance (Østergaard et al., 2015; Ueda et al., 2016). Furthermore, MALDI-TOF MS can be performed in a short time for a wide range pathogens in one experiment (Bizzini and Greub, 2010). Additionally, it does not need a high level of staff training, reducing the risk of laboratory-associated infections by minimizing handling of living culture materials needed for the preparation of isolates.

Thus, the aim of this study was to evaluate the efficacy of MALDI-TOF MS for the identification of four Gram-positive cocci, S. agalactiae, L. garvieae, S. iniae, and SDD isolated from the kidneys, brains or abscesses of diseased fish from different geographic locations between 2003 and 2016.

\section{MATERIALS AND METHODS}

\section{Bacterial Strains}

Bacterial strains were selected from the culture collection of the National Reference Laboratory for Aquatic Animal Diseases (AQUACEN) of the Brazilian Ministry of Agriculture, Livestock and Food Supply. These S. agalactiae $(n=50)$, L. garvieae $(n=11), S$. iniae $(n=47)$, and $\operatorname{SDD}(n=23)$ strains were isolated during bacteriological analyses of outbreaks in Brazilian fish farms in different years and geographical locations (Table S1). The isolation of these microorganisms was performed on chilled fish that were sent to AQUACEN for diagnosis. Swabs from brains, kidneys or abscesses were aseptically sampled and streaked onto $5 \%$ sheep blood agar (SBA) for the isolation of bacterial pathogens. These plates were incubated at $28^{\circ} \mathrm{C}$ for $48 \mathrm{~h}$. Finally, the identification of bacterial species was carried out as previously described (Mian et al., 2009; Netto et al., 2011; Figueiredo et al., 2012; Fukushima et al., 2017).

\section{Species Confirmation through 16S rRNA Gene Sequencing}

The isolates were thawed and streaked onto 5\% SBA and were incubated at $28^{\circ} \mathrm{C}$ for $48 \mathrm{~h}$. Isolates were incubated in a lysozyme solution at $37^{\circ} \mathrm{C}$ overnight. Bacterial DNA was extracted with a Maxwell 16 Tissue DNA purification kit (Promega, Madison, WI, USA) according to the manufacturer's instructions. The extracted DNA was quantified using a Nanodrop spectrophotometer (Thermo Scientific, Wilmington, DE, USA). The purity of the extracted DNA was determined using the absorbance ratio at $260 / 280 \mathrm{~nm}$. Samples with ratio of $1.8 \pm 0.5$ were stored at $-80^{\circ} \mathrm{C}$ until use.

The 16S rRNA gene was amplified by PCR with the universal primers B37 (5'-TAC GGY TAC CTT GTT ACG A-3') and C70 (5'-AGA GTT TGA TYM TGGC-3') and PCR amplicons were purified according to the method described by Fox et al. (1995) for all strains used in this work. The sequencing reactions were performed using a BigDye ${ }^{\mathrm{TM}}$ Terminator Cycle Sequencing Kit (Applied Biosystems, UK) and evaluated with an ABI 3,500 Genetic Analyzer (Life Technologies, USA). Forward and reverse sequencing products were used to generate contigs with the BioEdit software (Ibis Biosciences, Carlsbad, USA) version 7.2. Their identity was evaluated using the BLAST webserver (http:// www.ncbi.nlm.nih.gov/BLAST) by checking against existing sequences in the $\mathrm{nt} / \mathrm{nr}$ database. A similarity of $\geq 97 \%$ was considered as the same species in accordance with Nguyen et al. (2016) and Větrovský and Baldrian (2013).

\section{MALDI-TOF MS Real-Time Identification Analysis}

All isolates were thawed and streaked onto 5\% SBA and incubated at $28^{\circ} \mathrm{C}$ for $48 \mathrm{~h}$. A fresh, single colony of each bacterial strain was spotted using a toothpick into a target steel plate. For each strain, $1 \mu \mathrm{l}$ of formic acid (70\%) and $1 \mu \mathrm{l}$ of MALDI-TOF MS matrix, consisting of a saturated solution of $\alpha$-cyano-4-hydroxycinnamic acid (HCCA) (Bruker Daltonics, Bremen, Germany), were applied to the spot and allowed to air-dry. Spectra were acquired using the FlexControl MicroFlex LT mass spectrometer (Bruker Daltonics) with a $60-\mathrm{Hz}$ nitrogen laser, in which up to 240 laser shots are fired in spiral movements to collect 40 shot steps for each strain spot. Furthermore, parameters for mass range detection were defined to allow the identification from 1,960 to $20,137 \mathrm{~m} / \mathrm{z}$, where Ion source $1 \mathrm{v}$ was $19.99 \mathrm{kv}$, Ion source 2 voltage was $18.24 \mathrm{kv}$ and the lens voltage was 6.0 $\mathrm{kv}$ for data acquisition. Prior to measurements, calibration was preceded with a bacterial test standard (E. coli DH5 alpha; Bruker Daltonics). The Real Time (RT) identification score criteria used were those recommended by the manufacturer: score $\geq 2.000$ indicates a species-level identification, score $\geq 1.700$ and $<2.000$ indicates a genus-level identification, and a score $<1.700$ indicates no reliable identification. Comparisons between MALDI-TOF MS strain identifications and those of other techniques were performed with $\mathrm{R}$ software version 3.0.1 ( $\mathrm{R}$ Core Team, 2013) with the agreement rates determined by the Kappa coefficient. 


\section{Creation of a Custom Main Spectra Profile}

To identify possible $S$. iniae strains and to enhance the $S$. dysgalactiae discrimination at the subspecies-level in a MALDI Biotyper, Main Spectra Profiles (MSPs) were created with reference strains for each species. Fresh colonies of the $S$. iniae SI23 strain and the SDD SD64, SD92 and SD142 strains were extracted according Alatoom et al. (2011). Briefly, the strains were collected from the agar and added to $300 \mu$ of distilled water, followed by the addition of $900 \mu \mathrm{l}$ of ethanol. Two rounds of centrifugation for $2 \mathrm{~min}$ at $13,000 \mathrm{rpm}$ and the complete removal of supernatant was necessary to obtain dried pellets. The pellets were suspended in $50 \mu \mathrm{l}$ of formic acid (70\%) and vortexed. Finally, $50 \mu \mathrm{l}$ of acetonitrile was added and the mixtures were centrifuged for $2 \mathrm{~min}$ at 13,000 rpm. For assays, one microliter of the supernatant was spotted eight times onto a steel target. Directly after air-drying, each spot was overlaid with $1 \mu \mathrm{l}$ of HCCA matrix. Each spot was measured three times with the same protocol/parameters described in the section above. The obtained spectra were closely analyzed in the FlexAnalysis software (Bruker Daltonics) to assess the high level of reproducibility. Finally, the spectra of each strain were uploaded to the MALDI Biotyper software version 3 (Bruker Daltonics) and assembled to generate a Main Spectra Profile (MSP) for the strains using the BioTyper MSP creation standard method. All steps were done according to the manufacturer's recommendations.

A figure illustrating the SD64 spectra was generated using $\mathrm{R}$ software version 3.0.1 (R Core Team, 2013), using data exported from the FlexAnalysis software (Bruker Daltonics). In addition, in order to compare the custom MSPs with the MSP preloaded on the Bruker MSP library, the BioTyper software version 3.0 (Bruker Daltonics) was used to perform a dendrogram analysis. The parameters used were distance measure = "correlation," linkage $=$ "average," maximum number of top level nodes $=$ " 0 ," score oriented dendrogram "enabled," score threshold values for a single organism = "300," and score threshold values for a related organism $=$ “ 0 .”

\section{Streptococcus dysgalactiae Subspecies Confirmation}

The SDD strains that had subspecies suggested by Costa et al. (2014) were inferred by a BLAST comparison of the 16S rRNA and $\operatorname{sodA}$ genes, and the MALDI Biotyper (Bruker Daltonics) analysis suggested a closer relationship with $S$. dysgalactiae subsp. equisimilis (SDE). In addition to the $16 \mathrm{~S}$ sequencing described above, a Next-Generation Sequence (NGS) experiment was performed. Three strains (SD64, SD92, and SD142) with different pulse-field gel electrophoresis (PFGE) profiles described in previous work from our group (Costa et al., 2014) were sequenced. DNA from the SDD strain was isolated from an overnight culture using a Maxwell 16 tissue DNA purification kit using the Maxwell 16 system (both from Promega). Sequencing was conducted on the Ion Torrent Personal Genome Machine sequencing system (Life Technologies) using a 200 bp fragment library kit, according to the manufacturer's recommendations. The barcodes of the raw data were removed using an in-house script (https:/github.com/aquacen/fast_sample), and assembly was performed using SPAdes v3.9.1 (Nurk et al., 2013).
SDD taxonomic classification was determined using the Jensen and Kilian (2012) method, where the analysis of the phylogenetic relationship of seven housekeeping genes (map, $p f l, p p a C, p y k, r p o B, \operatorname{sod} A$, and tuf) through a multilocus sequence analysis (MLSA) represent an improved basis for the identification of clinically important streptococci. The concatenated sequence of these housekeeping genes is used to establish differences between species that allow a more accurate identification within the pyogenic group of streptococci. The sequence of the draft genome of SDD ATCC 27957 is available on GenBank (Accession number: CM001076) and together with the genes of 30 streptococci strains submitted with the work of Jensen and Kilian (2012) were downloaded (Accession numbers: map: JN632385 to JN632479; pfl: JN632290 to JN632384; ppaC: JN632195 to JN632289; pyk: JN632100 to JN632194; rpoB: JN632005 to JN632099; sodA: JN631910 to JN632004; tuf: JN631815 to JN631909).

To extract the sequences of the corresponding housekeeping genes, a homology search for each of the seven genes in the SD64, SD92, and SD142 strains was performed using the BLAST webserver (http://www.ncbi.nlm.nih.gov/BLAST), with contigs generated by assembly software. The same strategy was performed with the SDD ATCC 27957 strain. All genes for each strain were concatenated in the following order: mappfI-ppaC-pyk-rpoB-sodA-tuf. Alignment and phylogeny analyses were performed using MEGA6 (Tamura et al., 2013), with the Kimura-2 model parameters, using the Minimum Evolution algorithm, and a bootstrap of 1,000 replications.

\section{RESULTS}

\section{Species Confirmation through 16S rRNA Gene Sequencing}

The sequences of the 16S rRNA PCR products, which were generated with the aforementioned forward and reverse primers, were comprised in contigs for each strain. The mean lengths of the contigs were $1,514 \pm 12,1,537 \pm 14,1,519 \pm 15$, and $1,515 \pm 17$ bp for S. agalactiae, L. garvieae, S. iniae, and SDD, respectively. The contigs from each strain were used as queries for the BLAST webserver, and a percentage value of the similarities for L. garvieae was between 98 and 100, whereas S. agalactiae, S. iniae and SDD varied between 97 and 100. For the SDD strains, it was not possible make identification at the subspecies-level. For each SDD isolate there were results referring to the SDE and SDD with the same percentage value of identity that referred to the same query coverage.

\section{MALDI-TOF MS RT Identification of $S$. agalactiae and $L$. garvieae}

For each strain-spot, 1-3 spectra were expected, according to the manufacturer's instructions for quality assurance performed by MALDI Biotyper software of acquisition. For S. agalactiae, 64 spectra were acquired, whereas 11 spectra were acquired for L. garvieae. All strains for both species were identified at the species-level (score $\geq 2.000$ ). The minimal and maximal scores for S. agalactiae were 2.083 and 2.377 (Table 1), respectively, and for L. garvieae were 2.081 and 2.218 (Table 2), respectively. For 
TABLE 1 | Streptococcus agalactiae strains identification by $16 S$ rRNA sequencing and MALDI-TOF MS.

\begin{tabular}{|c|c|c|c|c|}
\hline \multirow[t]{2}{*}{ Strain } & \multicolumn{2}{|c|}{ 16S rRNA sequencing } & \multicolumn{2}{|l|}{ MALDI Biotyper } \\
\hline & Species & $\begin{array}{c}\% \\
\text { Identity }\end{array}$ & Organism best match & $\begin{array}{l}\text { Score } \\
\text { value }\end{array}$ \\
\hline SA001 & Streptococcus agalactiae & 100 & Streptococcus agalactiae & 2.330 \\
\hline SA005 & Streptococcus agalactiae & 100 & Streptococcus agalactiae & 2.318 \\
\hline SA007 & Streptococcus agalactiae & 100 & Streptococcus agalactiae & 2.371 \\
\hline SA009 & Streptococcus agalactiae & 100 & Streptococcus agalactiae & 2.302 \\
\hline SA016 & Streptococcus agalactiae & & Streptococcus agalactiae & 2.357 \\
\hline SA020 & Streptococcus agalactiae & 100 & Streptococcus agalactiae & 2.296 \\
\hline SA030 & Streptococcus agalactiae & 100 & Streptococcus agalactiae & 2.289 \\
\hline SA033 & Streptococcus agalactiae & 100 & Streptococcus agalactiae & 2.211 \\
\hline SA053 & Streptococcus agalactiae & 100 & Streptococcus agalactiae & 2.206 \\
\hline SA073 & Streptococcus agalactiae & 100 & Streptococcus agalactiae & 2.259 \\
\hline SA075 & Streptococcus agalactiae & 100 & Streptococcus agalactiae & 2.189 \\
\hline SA079 & Streptococcus agalactiae & 100 & Streptococcus agalactiae & 2.251 \\
\hline SA081 & Streptococcus agalactiae & 100 & Streptococcus agalactiae & 2.327 \\
\hline SA085 & Streptococcus agalactiae & 100 & Streptococcus agalactiae & 2.207 \\
\hline SA095 & Streptococcus agalactiae & 100 & Streptococcus agalactiae & 2.275 \\
\hline SA097 & Streptococcus agalactiae & 100 & Streptococcus agalactiae & 2.227 \\
\hline SA102 & Streptococcus agalactiae & 99 & Streptococcus agalactiae & 2.172 \\
\hline SA117 & Streptococcus agalactiae & 97 & Streptococcus agalactiae & 2.162 \\
\hline SA132 & Streptococcus agalactiae & 100 & Streptococcus agalactiae & 2.322 \\
\hline SA136 & Streptococcus agalactiae & 100 & Streptococcus agalactiae & 2.220 \\
\hline SA159 & Streptococcus agalactiae & 100 & Streptococcus agalactiae & 2.364 \\
\hline SA172 & Streptococcus agalactiae & 100 & Streptococcus agalactiae & 2.339 \\
\hline SA184 & Streptococcus agalactiae & 100 & Streptococcus agalactiae & 2.306 \\
\hline SA191 & Streptococcus agalactiae & 100 & Streptococcus agalactiae & 2.207 \\
\hline SA201 & Streptococcus agalactiae & 100 & Streptococcus agalactiae & 2.221 \\
\hline SA209 & Streptococcus agalactiae & 100 & Streptococcus agalactiae & 2.309 \\
\hline SA212 & Streptococcus agalactiae & 100 & Streptococcus agalactiae & 2.377 \\
\hline SA218 & Streptococcus agalactiae & 100 & Streptococcus agalactiae & 2.331 \\
\hline SA220 & Streptococcus agalactiae & 100 & Streptococcus agalactiae & 2.351 \\
\hline SA245 & Streptococcus agalactiae & 100 & Streptococcus agalactiae & 2.192 \\
\hline SA256 & Streptococcus agalactiae & 100 & Streptococcus agalact & 2.083 \\
\hline SA289 & Streptococcus agalactiae & 100 & Streptococcus agalactiae & 2.167 \\
\hline SA330 & Streptococcus agalactiae & 100 & Streptococcus agalactiae & 2.317 \\
\hline SA333 & Streptococcus agalactiae & 100 & Streptococcus agalactiae & 2.294 \\
\hline SA341 & Streptococcus agalactiae & 100 & Streptococcus agalactiae & 2.296 \\
\hline SA343 & Streptococcus agalactiae & 100 & Streptococcus agalactiae & 2.276 \\
\hline SA346 & Streptococcus agalactiae & 100 & Streptococcus agalactiae & 2.254 \\
\hline SA374 & Streptococcus agalactiae & 100 & Streptococcus agalactiae & 2.363 \\
\hline SA375 & Streptococcus agalactiae & 100 & Streptococcus agalactiae & 2.360 \\
\hline SA623 & Streptococcus agalactiae & 100 & Streptococcus agalactiae & 2.248 \\
\hline SA627 & Streptococcus agalactiae & 100 & Streptococcus agalactiae & 2.349 \\
\hline SA665 & Streptococcus agalactiae & 97 & Streptococcus agalactiae & 2.281 \\
\hline SA719 & Streptococcus agalactiae & 98 & Streptococcus agalactiae & 2.197 \\
\hline SA796 & Streptococcus agalactiae & 97 & Streptococcus agalactiae & 2.359 \\
\hline SA808 & Streptococcus agalactiae & 97 & Streptococcus agalactiae & 2.242 \\
\hline SA887 & Streptococcus agalactiae & 97 & Streptococcus agalactiae & 2.185 \\
\hline & Streptococcus agalactiae & 99 & Streptococcus agalactiae & \\
\hline SA941 & Streptococcus agalactiae & 97 & Streptococcus agalactiae & 2.257 \\
\hline SA959 & Streptococcus agalactiae & 97 & Streptococcus agalactiae & 2.328 \\
\hline SA972 & Streptococcus agalactiae & 97 & Streptococcus agalactiae & 2.183 \\
\hline
\end{tabular}

TABLE 2 | Lactococcus garvieae strains identification by 165 rRNA sequencing and MALDI-TOF MS.

\begin{tabular}{llcllll}
\hline Strain & \multicolumn{2}{c}{ 16S rRNA sequencing } & & \multicolumn{2}{c}{ MALDI Biotyper } \\
\cline { 2 - 3 } & Species & $\begin{array}{c}\% \\
\text { Identity }\end{array}$ & & Organism best match & $\begin{array}{c}\text { Score } \\
\text { value }\end{array}$ \\
\hline LG002 & Lactococcus garvieae & 100 & & Lactococcus garvieae & 2.166 \\
LG005 & Lactococcus garvieae & 99 & & Lactococcus garvieae & 2.195 \\
LG009 & Lactococcus garvieae & 98 & & Lactococcus garvieae & 2.084 \\
LG010 & Lactococcus garvieae & 98 & & Lactococcus garvieae & 2.218 \\
LG011 & Lactococcus garvieae & 100 & & Lactococcus garvieae & 2.213 \\
LG015 & Lactococcus garvieae & 100 & & Lactococcus garvieae & 2.142 \\
LG018 & Lactococcus garvieae & 99 & & Lactococcus garvieae & 2.110 \\
LG019 & Lactococcus garvieae & 98 & & Lactococcus garvieae & 2.114 \\
LG020 & Lactococcus garvieae & 100 & & Lactococcus garvieae & 2.184 \\
LG021 & Lactococcus garvieae & 99 & & Lactococcus garvieae & 2.165 \\
LG022 & Lactococcus garvieae & 98 & & Lactococcus garvieae & 2.081 \\
\hline
\end{tabular}

both species a perfect agreement (Kappa $=1$; CI: 1.0-1.0; and $p$ $<0.005)$ was observed between the 16S rRNA gene sequencing and MALDI-TOF MS techniques to identify the species.

\section{MALDI-TOF MS RT Identification of S. iniae}

A total of 52 spectra were obtained for the 47 strains. Identification of $S$. iniae was possible in $\sim 53 \%$ of isolates at the genus-level (Table 3 ), and the minimal and maximal scores were 1.482 and 1.854, respectively, including 22 with no reliable identification. The genus-level was inferred by an approximation of the spectra with $S$. dysgalactiae $(n=7)$, S. equi $(n=1)$, and $S$. pyogenes $(n=17)$. The species identification agreement when comparing $16 \mathrm{~S}$ rRNA gene sequencing and MALDI-TOF MS was poor (Kappa $=0.04$; CI: -0.03 to 0.11 ; and $p=0.063$ ).

To make possible the correct identification of $S$. iniae strains using the MALDI Biotyper, a custom MSP was created for this species (Figure 1; MSP available at http://www.renaqua.gov.br/ aquacen-msp-si/). Twenty-four spectra were collected for one isolate (SI23) by the Biotyper RTC program. The spectra were analyzed in the FlexAnalysis software to identify a high level of reproducibility, and all spectra were used to create the MSP. A dendrogram generated in BioTyper software (Figure 2) shows the SI23 strain as a single leaf between the $S$. pyogenes and $S$. dysgalactiae clades. After the inclusion of the custom MSP of $S$. iniae, all the strains were identified at the species-level (Table 3), and the minimal and maximal score values were 2.013 and 2.426 , respectively. A complete agreement between both tested techniques was observed (Kappa $=1$; CI: 1.0-1.0; and $p<0.005$ ) for species identification.

\section{MALDI-TOF MS RT Identification of SDD}

The identification of SDD isolates, using 25 spectra from 23 strains, was obtained by an approximation of $S$. dysgalactiae and SDE MSPs at the species-level. Minimal and maximal scores were 2.058 and 2.298 , respectively. Of all the SDD strains, 13 were identified with proximity to the subspecies equisimilis, and in 10 strains, there was no discrimination of subspecies 
TABLE 3 | Streptococcus iniae strains identification by $16 \mathrm{~S}$ rRNA sequencing and MALDI-TOF MS (before and after custom MSP inclusion).

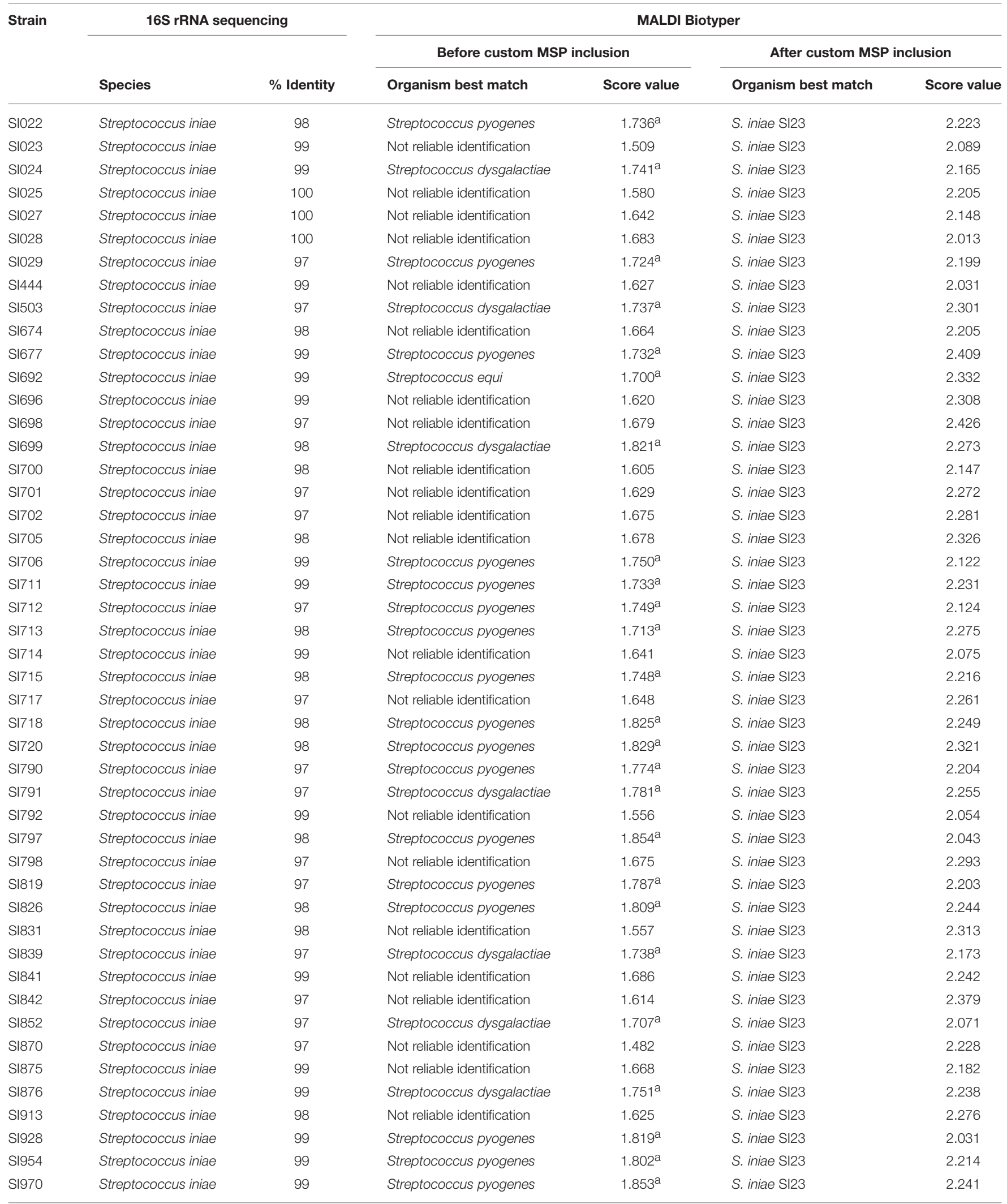

${ }^{a}$ Genus-level identification. 


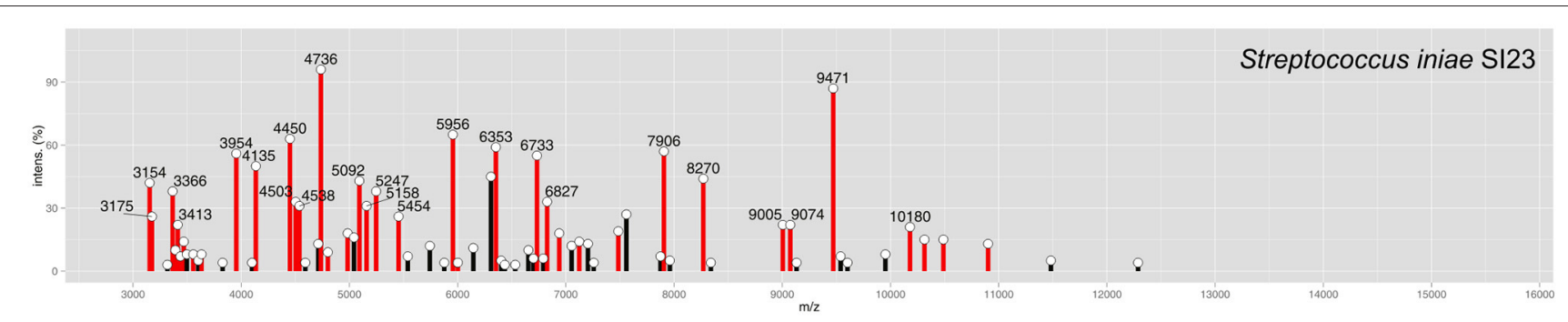

FIGURE 1 | MSP of S. iniae SI23 peak identification. Peaks with intensities greater than $20 \%$ are labeled. Peaks with a previously identified m/z (Kim et al., 2017) are shown in red bars.

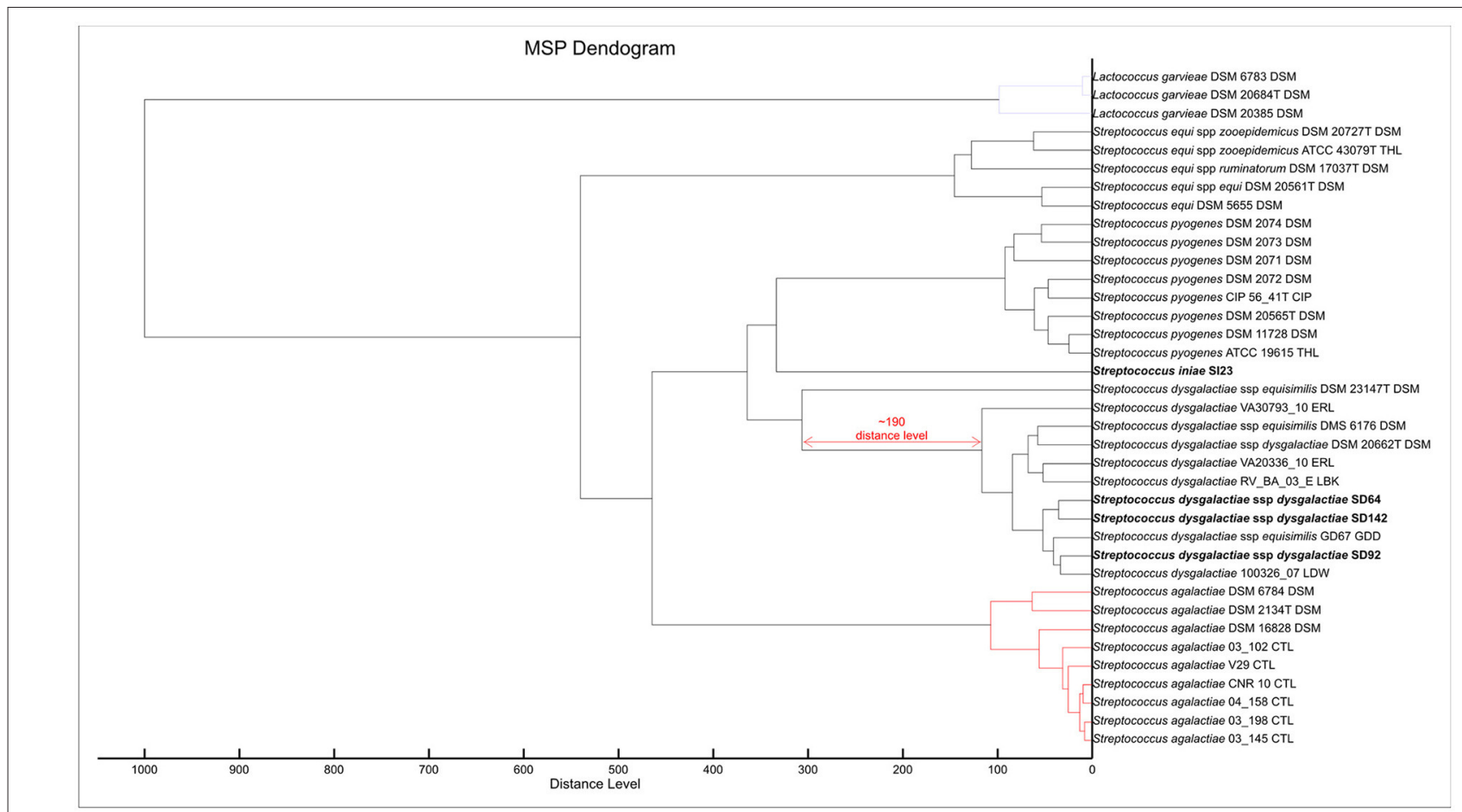

FIGURE 2 | MSP Dendrogram analysis of the custom MSP and Bruker MSP library of Lactococcus garvieae and Streptococcus spp. Isolates from this work are in bold. S. iniae SI23 is alone and between the S. pyogenes and S. dysgalactiae spp. clades. SDD is in an intra-species-specific clade of S. dysgalactiae spp. strains. The red arrow shows a distance level of $\sim 190$ from S. dysgalactiae spp. equisimilis DSM $23147^{\top}$ to other S. dysgalactiae strains.

(Table 4). The agreement between techniques was perfect when considering the species-level (Kappa $=1$; CI: 1.0-1.0; and $p<$ $0.004)$, but when considering the subspecies-level the agreement was only fair (Kappa $=0.21$; CI: $-0.08-0.52 ; p=0.075$ ). This demonstrated that both techniques were unable to identify strains at the subspecies-level.

These strains, according to previous work of our group (Costa et al., 2014), are from SDD subspecies. Therefore, an NGS experiment was done to confirm the subspecies assignments. Contigs from the assembly of the strains SD64, SD92, and SD142 (data not shown) were used for a MLSA analysis. The three strains formed a clade with SDD from work of Jensen and Kilian (2012), confirming the classification of theses strains as SDD subspecies in accordance with the methodology used (Figure 3).

To improve the identification by the MALDI Biotyper, custom MSPs were created for SDD (Figure 4; MSP available at http:// www.renaqua.gov.br/aquacen-msp-sdd/). Twenty-four spectra were collected for each isolate as described above and the spectra were analyzed in the FlexAnalysis, where all spectra were used to create the MSP. A dendrogram generated in BioTyper software (Figure 2) shows the SD64, SD92, and SD142 strains in an intra-species-specific clade of $S$. dysgalactiae spp. Figure 4 shows the common and exclusive peaks of custom MSPs and Bruker library MSPs, and, interestingly, the SDE DSM $23147^{\mathrm{T}}$ shows 25 exclusive peaks.

After this inclusion (Table 4), all isolates matched with to the three included custom MSP for the three best matches (Table S2), with minimal and maximal scores of 2.277 and 2.579, respectively. The agreement between $16 \mathrm{~S}$ rRNA gene sequencing and MALDI-TOF MS was poor (Kappa $=0.08, \mathrm{CI}:-0.05-0.22 ; p$ $=0.050$ ), considering that $16 \mathrm{~S}$ rRNA gene sequencing was unable to identify subspecies, whereas with MALDI-TOF MS, they could 


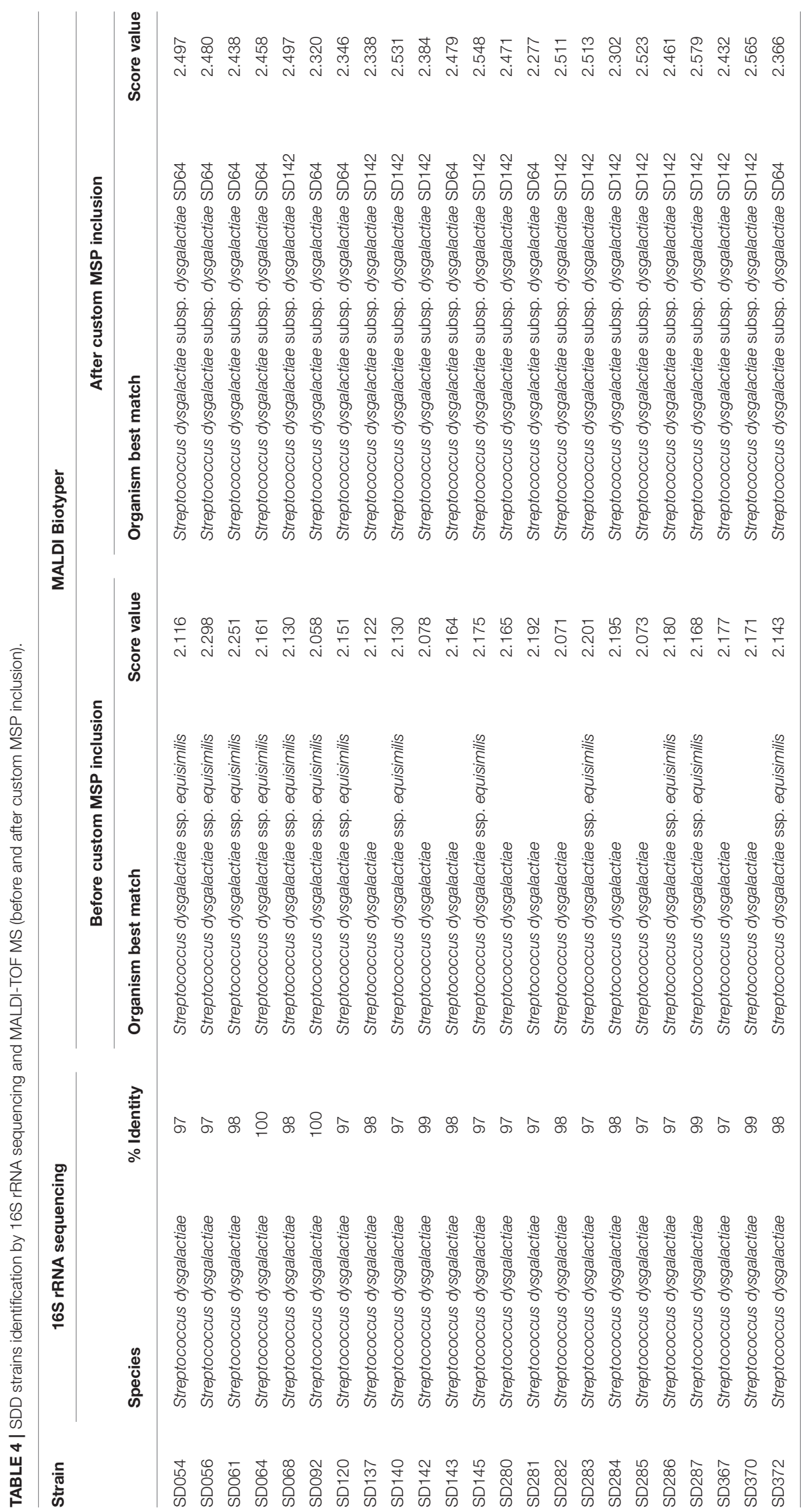




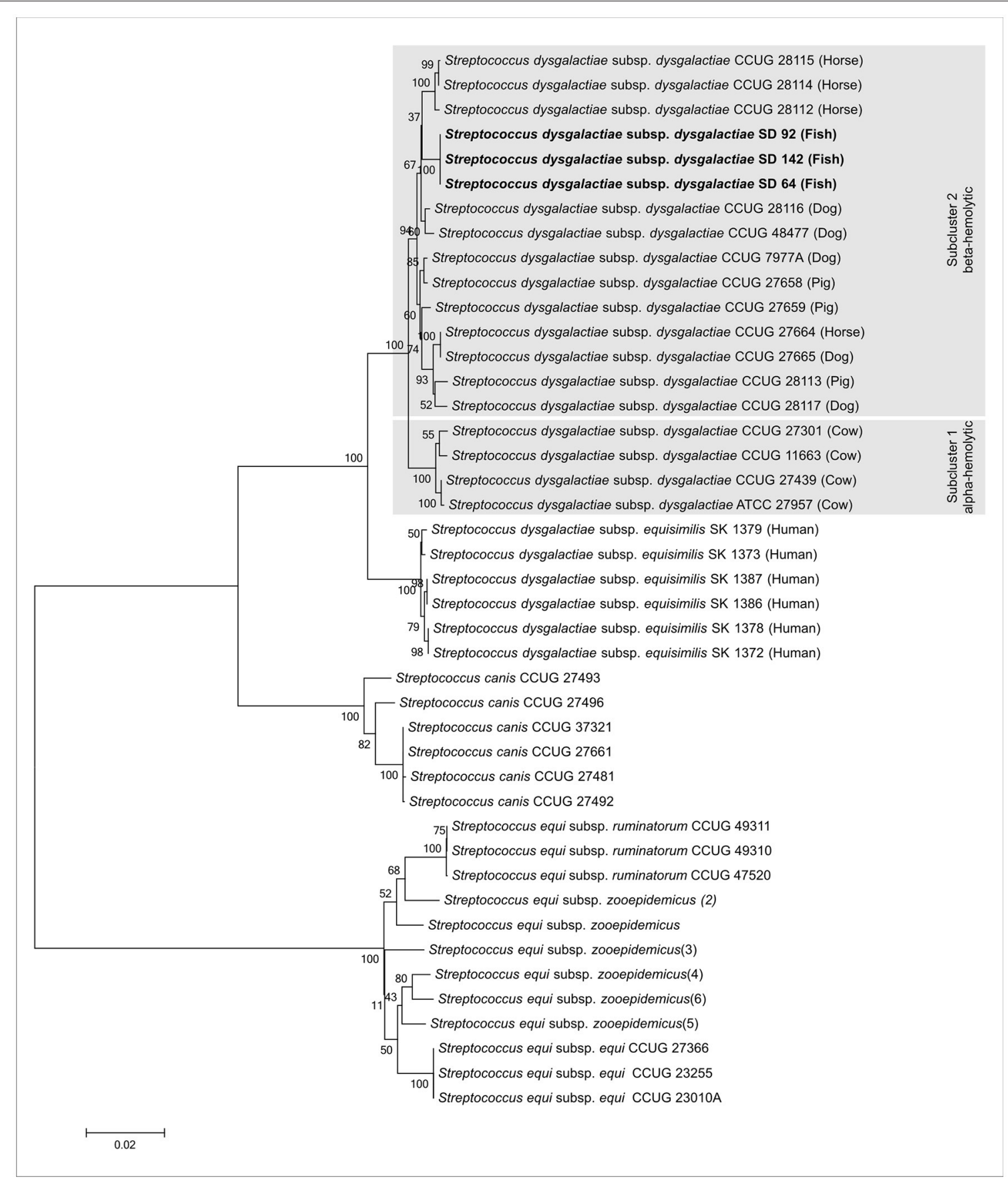

FIGURE 3 | Tree taxonomy analysis of SDD SD64, SD92, and SD142 strains. The strains from this work (bold) form a specific clade with other SDD strains from Jensen and Kilian (2012). Gray hatched areas are the subcluster of alpha- and beta-hemolytic strains proposed by Jensen and Kilian (2012).

be determined effectively. In contrast, considering the MLSA analysis, the agreement between this technique and MALDI-TOF MS was perfect (Kappa $=1$; CI: $1.0-1.0$; and $p<0.005)$.

\section{DISCUSSION}

Gram-positive cocci have been associated with acute and chronic fish diseases. They have become an increasingly important problem in the aquaculture industry in many countries (Evans et al., 2002; Vendrell et al., 2006; Agnew and Barnes, 2007; Mian et al., 2009; Netto et al., 2011; Figueiredo et al., 2012; Abdelsalam et al., 2013; Costa et al., 2014). An barrier to the better utilization of fish produced are the infectious diseases, including the control of the potential zoonotic infections caused by S. iniae (Keirstead et al., 2014). Thus, accelerating the diagnosis of diseases remains a big challenge. An alternative 

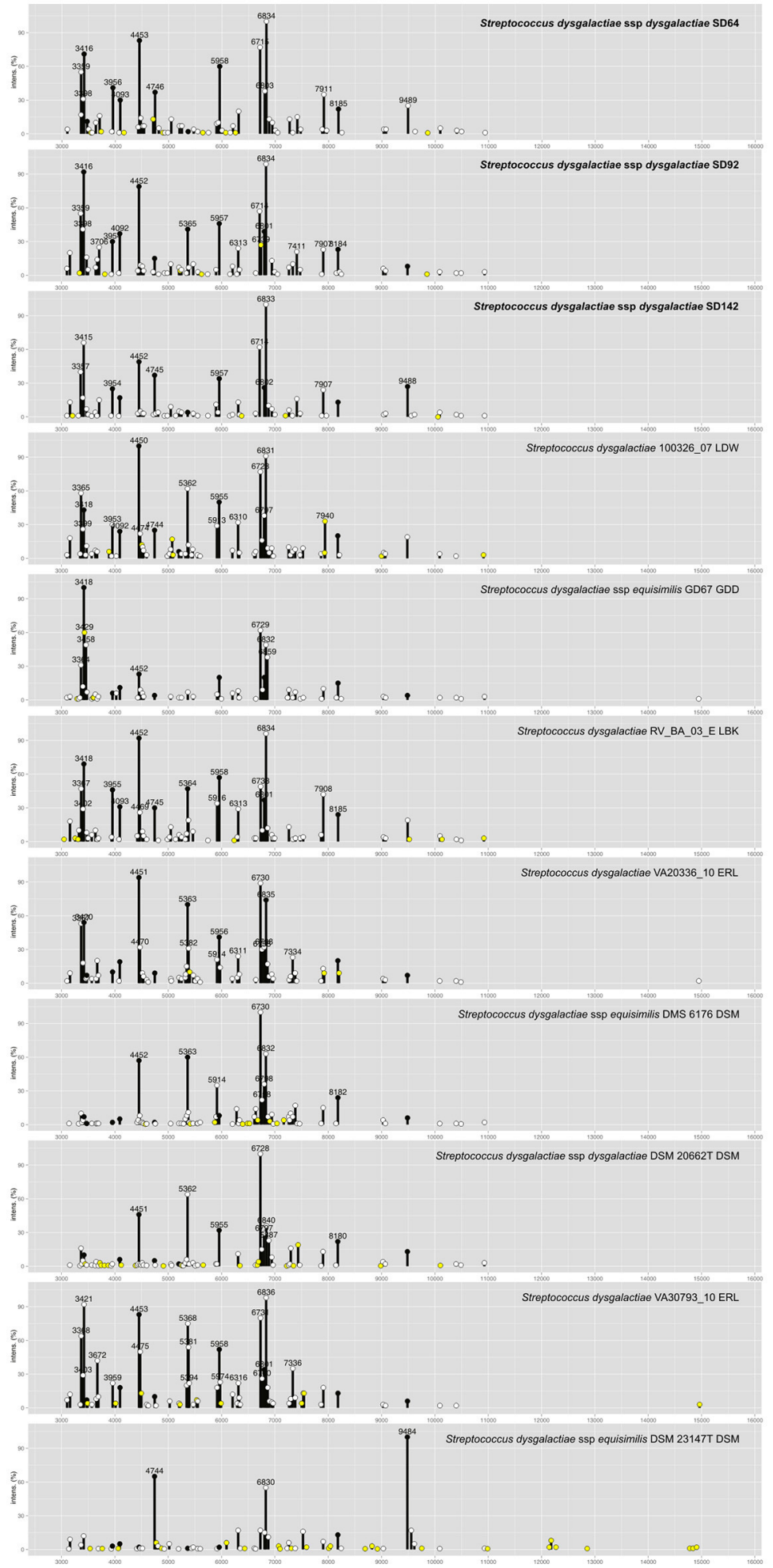

FIGURE 4 | Main Spectra Profiles of S. dysgalactiae subsp. dysgalactiae SD64, SD92, and SD142 peaks identification. The strains of this work (bold) together with the S. dysgalactiae group from Bruker MSP library. Peaks with intensities greater than $20 \%$ are labeled. Peaks common of all MSP are plotted in black circles. Peaks common to two or more MSPs are plotted in white circles. Peaks exclusive of each MSP are plotted in yellow circles. 
for these diagnoses is species-specific PCR and 16S rRNA gene sequencing, but these techniques are expensive, time consuming and require highly technical skills. Meanwhile, the MALDITOF MS method can be an important technique to increase the laboratory speeds of identification of the etiological agent because it is an efficient and cost-effective method for the rapid and routine identification of bacterial isolates in the clinical microbiology laboratory (Seng et al., 2009; Seibold et al., 2010). The potential for identification at the serotype or strain level, and antibiotic resistance profiling within minutes, makes MALDITOF MS an on-going revolution in the clinical microbiology laboratory (Romero-Gómez et al., 2012; Østergaard et al., 2015; Sauget et al., 2016; Ueda et al., 2016).

Streptococcus agalactiae and Lactococcus garvieae strains were classified as the correct species in $100 \%$ of the MALDI Biotyper experiments. Both species had been cited in previous works with MALDI-TOF MS systems (Lartigue et al., 2009; Navas et al., 2013), but not with regards to strains isolated from fish. Although there are no studies about the variation of the subtype of $L$. garvieae, a large number of $S$. agalactiae subtypes are known (Jones et al., 2003). The strains obtained from fish farm outbreaks in Brazil, used in this work, are from different genomic subtypes (Godoy et al., 2013), but nevertheless they did not show divergence in RT identification using the MALDI Biotyper.

The possibility of inclusion of a custom MSP on the Bruker MALDI Biotyper makes the tool expansive and allows for its adaptation to the laboratory business independent of the equipment manufacturer. Following the example of what had previously been reported by Segawa et al. (2015), the S. iniae SI23 strain and SDD SD64, SD92, and SD142 strains were included as MSPs, and the results improved to $100 \%$ correct identification. Recently, Fan et al. (2017), analyzing studies performed of streptococci rapid classification, suggested an overestimated accuracy of MALDI-TOF MS systems on Streptococcus spp. identification, since the $16 \mathrm{~S}$ rRNA gene sequencing analyses were only performed on discrepant results. In our analysis, all strains were identified by $16 \mathrm{~S}$ rRNA gene sequencing or by the $16 \mathrm{~S}$ rRNA gene in addition to housekeeping genes that were sequenced in parallel with the MALDI-TOF MS experiments, in order to achieve more confident results.

Streptococcus iniae strains, before the inclusion of a custom MSP, had matches with $S$. pyogenes and $S$. dysgalactiae, with scores lower than 2.000, suggesting a genus-level match (Table 3) within only $\sim 53 \%$ of tested isolates. The Bruker MSP library does not give MSP information about this species. The included custom MSP of SI23 showed similarities with these two species (Figure 2). These data corroborate with recent work from Kim et al. (2017) that shows the inclusion of S. iniae MSPs for the classification of $S$. iniae at the species-level, and shows the peaks list shared by $S$. iniae and S. pyogenes. Furthermore, 24 of 26 $(\sim 92 \%)$ of peaks with relative intensities greater than 20 are shared between S. iniae ATCC 29178 (Kim et al., 2017) and S. iniae SI23 (Figure 1).

In relation to the SDD strains, during the strains' RT classification, the results were all above 2.000; however, 13 strains were classified as SDE, and the other 10 were classified as
S. dysgalactiae species (Table 4). In previous work from our group (Costa et al., 2014), we suggested that the Brazilian S. dysgalactiae isolates were from a dysgalactiae subspecies, according to $16 \mathrm{~S}$ rRNA and $\operatorname{sod} A$ genes sequencing. Because of previous work (Jensen and Kilian, 2012) based on the MLSA analysis of a combination of seven housekeeping genes and the study of their phylogenetic relationships, an identification of the tested isolates in this work as SDD was confirmed. A custom MSP was created with the chosen isolates SD64, SD92, and SD142. Each strain has a different genotype that was identified in analyses made by PFGE in a previous work from our group (Costa et al., 2014). Using the custom MSP, all the analyzed strains had a correspondence larger than 2.000 (Table 4), indicating a high similarity of these strains with the created MSPs. Specimens in the Bruker MSP library named SDE and S. dysgalactiae do not have an accessible history, and the strain identified as SDD is referenced as ATCC $^{\circledR} 43078^{\mathrm{TM}}$, which is an isolate from a cow with mastitis (Garvie et al., 1983). Furthermore, as Figure 2 shows, the SDE DSM $23147^{T}$ showed a distance level (i.e., similarity of selected isolates with a maximal value of divergence of 1,000 ) of $\sim 190$ from another clade of $S$. dysgalactiae isolates and a different partner using MSP profiles in Figure 4. This characteristic suggests, taking into consideration there is no traceable information for the isolates in addition to the recent studies of $S$. dysgalactiae spp. (Jensen and Kilian, 2012; Ciszewski et al., 2016), that a reclassification, based on genomic analyses, should be done for such isolates from the Bruker MSP library.

Although the MALDI Biotyper is primarily designed for diagnoses at the species-level, in our experiments it was possible to correctly identify the subspecies of SDD, allowing for a rapid and low cost analysis when compared with other techniques to make subspecies-level identifications. MALDI-TOF MS was shown to be an efficient technology for identifying important Gram-positive cocci that cause major diseases in farmed fish.

\section{AUTHOR CONTRIBUTIONS}

GA, FP, and AZ wrote the manuscript. HF, GA, FP, GT, and CL conceived and designed the experiments. FP and AZ perform bioinformatics analyses. HF coordinated all analyses of the project. All authors read and approved the final manuscript.

\section{ACKNOWLEDGMENTS}

We thank the Coordination for the Improvement of Higher Education Personnel (CAPES), the National Counsel of Technological and Scientific Development (CNPq) and Ministry of Agriculture, Livestock, and Food Supply (MAPA) for financing this study.

\section{SUPPLEMENTARY MATERIAL}

The Supplementary Material for this article can be found online at: http://journal.frontiersin.org/article/10.3389/fmicb. 2017.01492/full\#supplementary-material 


\section{REFERENCES}

Abdelsalam, M., Asheg, A., and Eissa, A. E. (2013). Streptococcus dysgalactiae: an emerging pathogen of fishes and mammals. Int. J. Veter. Sci. Med. 1, 1-6. doi: 10.1016/j.ijvsm.2013.04.002

Agnew, W., and Barnes, A. C. (2007). Streptococcus iniae: an aquatic pathogen of global veterinary significance and a challenging candidate for reliable vaccination. Vet. Microbiol. 122, 1-15. doi: 10.1016/j.vetmic.2007.03.002

Alatoom, A. A., Cunningham, S. A., Ihde, S. M., Mandrekar, J., and Patel, R. (2011). Comparison of direct colony method versus extraction method for identification of gram-positive cocci by use of bruker biotyper matrix-assisted laser desorption ionization-time of flight mass spectrometry. J. Clin. Microb. 49, 2868-2873. doi: 10.1128/JCM.00506-11

Assis, G. B. N., Tavares, G. C., Pereira, F. L., Figueiredo, H. C. P., and Leal, C. A. G. (2016). Natural coinfection by Streptococcus agalactiae and Francisella noatunensis subsp. orientalis in farmed Nile tilapia (Oreochromis niloticus L.). J. Fish Dis. 40, 51-63. doi: 10.1111/jfd.12493

Bilecen, K., Yaman, G., Ciftci, U., and Laleli, Y. R. (2015). Performances and reliability of bruker microflex LT and VITEK MS MALDI-TOF mass spectrometry systems for the identification of clinical microorganisms. Biomed Res. Int. 2015:516410. doi: 10.1155/2015/516410

Bizzini, A., and Greub, G. (2010). Matrix-assisted laser desorption ionization timeof-flight mass spectrometry, a revolution in clinical microbial identification. Clin. Microbiol. Infect. 16, 1614-1619. doi: 10.1111/j.1469-0691.2010.03311.x

Brigante, G., Luzzaro, F., Bettaccini, A., Lombardi, G., Meacci, F., Pini, B., et al. (2006). Use of the phoenix automated system for identification of Streptococcus and Enterococcus spp. J. Clin. Microbiol. 44, 3263-3267. doi: 10.1128/JCM.00299-06

Ciszewski, M., Zegarski, K., and Szewczyk, E. M. (2016). Streptococcus dysgalactiae subsp. equisimilis isolated from infections in dogs and humans: are current subspecies identification criteria accurate? Curr. Microbiol. 73, 684-688. doi: 10.1007/s00284-016-1113-x

Clark, A. E., Kaleta, E. J., Arora, A., and Wolk, D. M. (2013). Matrix-assisted laser desorption ionization-time of flight massspectrometry: a fundamental shift in the routine practice of clinical microbiology. Clin. Microbiol. Rev. 26, 547-603. doi: 10.1128/CMR.00072-12

Clarridge, J. E. (2004). Impact of 16S rRNA gene sequence analysis for identification of bacteria on clinical microbiology and infectious diseases. Clin. Microbiol. Rev. 17, 840-862. doi: 10.1128/CMR.17.4.840-862.2004

Costa, F. A. A., Leal, C. A. G., Leite, R. C., and Figueiredo, H. C. P. (2014). Genotyping of Streptococcus dysgalactiae strains isolated from Nile tilapia, Oreochromis niloticus (L.). J. Fish Dis. 37, 463-469. doi: 10.1111/jfd.12125

Eldar, A., Bejerano, Y., Livoff, A., Horovicz, A., and Bercovier, H. (1995). Experimental streptococcal meningo-encephalitis in cultured fish. Vet. Microbiol. 43, 33-40. doi: 10.1016/0378-1135(94)00052-X

Evans, J. J., Klesius, P. H., Gilbert, P. M., Shoemaker, C. A., Sarawi, M. A. A., Landsberg, J., et al. (2002). Characterization of $\beta$-haemolytic Group B Streptococcus agalactiae in cultured seabream, Sparus auratus L., and wild mullet, Liza klunzingeri (Day), in Kuwait. J. Fish Dis. 25, 505-513. doi: 10.1046/j.1365-2761.2002.00392.x

Fan, W. T., Qin, T. T., Bi, R. R., Kang, H. Q., Ma, P., and Gu, B. (2017). Performance of the matrix-assisted laser desorption ionization time-of-flight mass spectrometry system for rapid identification of streptococci: a review. Eur. J. Clin. Microbiol. Infect. Dis. 36, 1005-1012. doi: 10.1007/s10096-016-2879-2

FAO (2016). The State of World Fisheries and Aquaculture (SOFIA). Available online at: http://www.fao.org/3/a-i5555e.pdf

Figueiredo, H. C. P., Netto, L. N., Leal, C. A. G., Pereira, U. P., and Mian, G. F. (2012). Streptococcus iniae outbreaks in Brazilian Nile tilapia (Oreochromis niloticus L.) farms. Braz. J. Microbiol. 43, 576-580. doi: 10.1590/S1517-83822012000200019

Fox, J. G., Yan, L. L., Dewhirst, F. E., Paster, B. J., Shames, B., Murphy, J. C., et al. (1995). Helicobacter bilis sp. Nov., A novel Helicobacter species isolated from bile, livers, and intestines of aged, inbred mice. J. Clin. Microbiol. 33, 445-454.

Fukushima, H. C. S., Leal, C. A. G., Cavalcante, R. B., Figueiredo, H. C. P., Arijo, S., Moriñigo, M. A., et al. (2017). Lactococcus garvieae outbreaks in Brazilian farms Lactococcosis in Pseudoplatystoma sp. - development of an autogenous vaccine as a control strategy. J. Fish Dis. 40, 263-272. doi: 10.1111/jfd.12509
Garvie, E. I., Farrow, J. A. E., and Bramley, A. J. (1983). Streptococcus dysgalactiae (Diernhofer) nom. rev. Int. J. Syst. Bacteriol. 33, 404-405. doi: 10.1099/00207713-33-2-404

Godoy, D. T., Carvalho-Castro, G. A., Leal, C. A. G., Pereira, U. P., Leite, R. C., and Figueiredo, H. C. P. (2013). Genetic diversity and new genotyping scheme for fish pathogenic Streptococcus agalactiae. Lett. Appl. Microbiol. 57, 476-483. doi: 10.1111/lam.12138

Jensen, A., and Kilian, M. (2012). Delineation of Streptococcus dysgalactiae, its subspecies, and its clinical and phylogenetic relationship to Streptococcus pyogenes. J. Clin. Microbiol. 50, 112-126. doi: 10.1128/JCM.05900-11

Jones, N., Bohnsack, J. F., Takahashi, S., Oliver, K. A., Chan, M. S., Kunst, F., et al. (2003). Multilocus sequence typing system for group B streptococcus. J. Clin. Microbiol. 6, 2530-2536. doi: 10.1128/JCM.41.6.2530-2536.2003

Keirstead, N. D., Brake, J. W., Griffin, M. J., Halliday-Simmonds, I., Thrall, M. A., and Soto, E. (2014). Fatal Septicemia caused by the Zoonotic Bacterium Streptococcus iniae during an outbreak in Caribbean reef fish. Vet. Pathol. 51, 1035-1041. doi: 10.1177/0300985813505876

Kim, S. W., Nho, S. W., Im, S. P., Lee, J. S., Jung, J. W., Lazarte, J. M., et al. (2017). Rapid MALDI biotyper-based identification and cluster analysis of Streptococcus iniae. J. Microbiol. 55, 260-266. doi: 10.1007/s12275-017-6472-x

Kolbert, C. P., and Persing, D. H. (1999). Ribosomal DNA sequencing as a tool for identification of bacterial pathogens. Curr. Opin. Microbiol. 2, 299-305. doi: 10.1016/S1369-5274(99)80052-6

Lartigue, M. F., Héry-Arnaud, G., Haguenoer, E., Domelier, A. S., Schmit, P. O., van der Mee-Marquet, N., et al. (2009). Identification of Streptococcus agalactiae isolates from various phylogenetic lineages by matrix-assisted laser desorption ionization-time of flight mass spectrometry. J. Clin. Microbiol. 47, 2284-2287. doi: 10.1128/JCM.00175-09

Mian, G. F., Godoy, D. T., Leal, C. A. G., Yuhara, T. Y., Costa, G. M., and Figueiredo, H. C. P. (2009). Aspects of the natural history and virulence of S. agalactiae infection in Nile tilapia. Vet. Microbiol. 36, 180-183. doi: 10.1016/j.vetmic.2008.10.016

Nagy, E., Urbán, E., and Nord, C. E. (2011). ESCMID study group on antimicrobial resistance in Anaerobic Bacteria 2011 antimicrobial susceptibility of Bacteroides fragilis group isolates in Europe: 20 years of experience. Clin. Microbiol. Infect. 17, 371-379. doi: 10.1111/j.1469-0691.2010.03256.x

Navas, M. E., Hall, G., and El Bejjani, D. (2013). A case of Endocarditis caused by Lactococcus garvieae and suggested methods for identification. J. Clin. Microbiol. 51, 1990-1992. doi: 10.1128/JCM.03400-12

Netto, L. N., Leal, C. A. G., and Figueiredo, H. C. P. (2011). Streptococcus dysgalactiae as an agent of septicaemia in Nile tilapia, Oreochromis niloticus (L.). J. Fish Dis. 34, 251-254. doi: 10.1111/j.1365-2761.2010.01220.x

Nguyen, N. P., Warnow, T., Pop, M., and White, B. (2016). A perspective on 16S rRNA operational taxonomic unit clustering using sequence similarity. Npj Biofilms Microbiomes. 2:16004. doi: 10.1038/npjbiofilms.2016.4

Nomoto, R., Unose, N., Shimahara, Y., Nakamura, A., Hirae, T., Maebuchi, K., et al. (2006). Characterization of lancefield group C Streptococcus dysgalactiae isolated from farmed fish. J. Fish Dis. 29, 673-682. doi: 10.1111/j.1365-2761.2006.00763.x

Nurk, S., Bankevich, A., Antipov, D., Gurevich, A., Korobeynikov, A., Lapidus, A., et al. (2013). Assembling genomes and mini-metagenomes from highly chimeric reads. Lect. Notes Comput. Sci. 20, 714-737. doi: 10.1007/978-3-642-37195-0_13

Østergaard, C., Hansen, S. G. K., and Møller, J. K. (2015). Rapid firstline discrimination of methicillin resistant Staphylococcus aureus strains using MALDI-TOF MS. Int. J. Med. Microbiol. 305, 838-847. doi: 10.1016/j.ijmm.2015.08.002

Patel, J. B. (2001). 16S rRNA gene sequencing for bacterial pathogen identification in the clinical laboratory. Mol. Diagn. 6, 313-321. doi: 10.2165/00066982-200106040-00012

Poyart, C., Quesne, G., Coulon, S., Berche, P., and Trieu-Cuot, P. (1998). Identification of streptococci to species level by sequencing the gene encoding the manganese-dependent superoxide dismutase. J. Clin. Microbiol. 36, 41-47.

R Core Team (2013). R: A Language and Environment for Statistical Computing. Vienna: R Foundation for Statistical Computing.

Rizzardi, K., Wahab, T., and Jernberg, C. (2013). Rapid subtyping of Yersinia enterocolitica by matrix-assisted laserdesorption ionization-time of flight mass 
spectrometry (MALDI-TOF MS) for diagnostics and surveillance. J. Clin. Microbiol. 51, 4200-4203. doi: 10.1128/JCM.01416-13

Romero-Gómez, M. P., Gómez-Gil, R., Paño-Pardo, J. R., and Mingorance, J. (2012). Identification and susceptibility testing of microorganism by direct inoculation from positive blood culture bottles by combining MALDITOF and Vitek-2 Compact is rapid and effective. J. Infect. 65, 513-520. doi: 10.1016/j.jinf.2012.08.013

Sauget, M., van der Mee-Marquet, N., Bertrand, X., and Hocquet, D. (2016). Matrix-assisted laser desorption ionization-time of flight Mass spectrometry can detect Staphylococcus aureus clonal complex 398. J. Microbiol. Methods. 127, 20-23. doi: 10.1016/j.mimet.2016.05.010

Segawa, S., Nishimura, M., Sogawa, K., Tsuchida, S., Murata, S., Watanabe, M., et al. (2015). Identification of Nocardia species using matrix-assisted laser desorption/ionization-time-of-flight mass spectrometry. Clin. Proteomics. 12:6. doi: 10.1186/s12014-015-9078-5

Seibold, E., Maier, T., Kostrzewa, M., Zeman, E., and Splettstoesser, W. (2010). Identification of Francisella tularensis by whole-cell matrix-assisted laser desorption ionization-time of flight mass spectrometry: fast, reliable, robust, and cost-effective differentiation on species and subspecies levels. J. Clin. Microbiol. 48, 1061-1069. doi: 10.1128/JCM.01953-09

Seng, P., Drancourt, M., Gouriet, F., Scola, B., Fournier, P., Rolain, J. M., et al. (2009). Ongoing revolution in bacteriology: routine identification of bacteria by matrix-assisted laser desorption ionization time-of-flight mass spectrometry. Clin. Infect. Dis. 49, 543-551. doi: 10.1086/600885

Singhal, N., Kumar, M., Kanaujia, P. K., and Virdi, J. S. (2015). MALDI-TOF mass spectrometry: an emerging technology for microbial identification and diagnosis. Front. Microbiol. 6:791. doi: 10.3389/fmicb.2015.00791

Soto, E., Wang, R., Wiles, J., Baumgartner, W., Green, C., Plumb, J., et al. (2015). Characterization of isolates of Streptococcus agalactiae from diseased farmed and wild marine fish from the U.S. Gulf Coast, Latin America, and Thailand. J. Aquat. Anim. Health. 27, 123-134. doi: 10.1080/08997659.2015.1032439
Tamura, K., Stecher, G., Peterson, D., Filipski, A., and Kumar, S. (2013). MEGA6: molecular evolutionary genetics analysis version 6.0. Mol. Biol. Evol. 30, 2725-2729. doi: 10.1093/molbev/mst197

Tavares, G. C., Costa, F. A. A., Santos, R. R., Barony, G. M., Leal, C. A. G., and Figueiredo, H. C. P. (2016). Nonlethal sampling methods for diagnosis of Streptococcus agalactiae infection in Nile tilapia, Oreochromis niloticus (L.). Aquaculture 454, 237-242. doi: 10.1016/j.aquaculture.2015. 12.028

Ueda, O., Tanaka, S., Nagasawa, Z., Hanaki, H., Shobuike, T., and Miyamoto, H. (2016). Development of a novel matrix-assisted laser desorption/ionization time-of-flight mass spectrum (MALDI-TOF-MS)-based typing method to identify meticillin-resistant Staphylococcus aureus clones. J. Hosp. Infect. 90, 147-155. doi: 10.1016/j.jhin.2014.11.025

Vendrell, D., Balcázar, J. L., Ruiz-Zarzuela, I., Blas, I., Gironés, O., and Múzquiz, L. (2006). Lactococcus garvieae in fish: a review. Comp. Immunol. Microbiol. Infect. Dis. 29, 177-198. doi: 10.1016/j.cimid.2006.06.003

Větrovský, T., and Baldrian, P. (2013). The variability of the 16S rRNA gene in bacterial genomes and its consequences for bacterial community analyses. PLoS ONE 8:e57923. doi: 10.1371/journal.pone.0057923

Conflict of Interest Statement: The authors declare that the research was conducted in the absence of any commercial or financial relationships that could be construed as a potential conflict of interest.

Copyright $\odot 2017$ Assis, Pereira, Zegarra, Tavares, Leal and Figueiredo. This is an open-access article distributed under the terms of the Creative Commons Attribution License (CC BY). The use, distribution or reproduction in other forums is permitted, provided the original author(s) or licensor are credited and that the original publication in this journal is cited, in accordance with accepted academic practice. No use, distribution or reproduction is permitted which does not comply with these terms. 